\title{
An Extensive Search for Overtones in Schwarzschild Black Holes
}

\author{
E. Abdalla and D. Giugno \\ Instituto de Física, Universidade de São Paulo \\ C.P. 66318, CEP 05315, São Paulo-SP, Brazil
}

Received on 2 April, 2007

\begin{abstract}
In this paper we show that with standard numerical methods it is possible to obtain highly precise results for quasi normal modes (QNMs). In particular, secondary modes are obtained by numerical integration done in the well-known null grid. We have compared such numerical results to the also well-known 6th-order WKB method and have found a striking degree of agreement, which could be as good as seven significant figures for the fundamental mode and three for the first overtone. We have chosen the Schwarzschild BH (black hole) to start with because it is the simplest and most well-known of all BHs, so it provides a very safe testing ground to the aforementioned numerical method.
\end{abstract}

Keywords: Gravitation; Perturbations; Neutron Stars; Quark Stars

\section{INTRODUCTION}

Wave propagation around black holes is an active field of research (see [1],[2]). The perspective of gravitational wave detection in a near future and the great recent development of numerical general relativity have increased even further the activity on this field [3]. Gravitational waves should be especially strong when emitted by black holes. The study of the propagation of perturbations around them is, hence, essential to provide templates for the gravitational wave identification. A recent challenge has been posed by advances in the black hole merger problem, in which the ringdown phase characterisation from the numerical wave profile is poorly understood, so that the techniques here employed and discussed may be of interest in that context. Thus, activity in this field is developing quickly [1],[4].

The Schwarzschild black hole is very well known $[2,5]$ but it is important to get reassured about the robustness of the methods and results. Therefore, here, we embark in a detailed study of the secondary modes by means of the subtraction of the first modes in the time domain. The method, though very simple has not been used in the literature due to numerical errors intrinsic to the method. However, we were able to use simple standard methods to show that the results are valid implying an acuracy of up to seven figures for the dominant (fundamental) mode and sometimes three for the secondary mode (or first overtone).

In what follows, we have used the geometric system of units, for which $c=G=1$. This means that the masses have dimension of length. The conversion factor to CGS/MKS units is $c^{2} / G$.

\section{FUNDAMENTAL MODES AND FIRST OVERTONES}

We begin with a series of tables on the frequencies of the QNMs for the Schwarzschild black holes, for the scalar, electromagnetic (EM) and axial gravitational perturbations. We have employed, throughout our discussion, $M=1.0$, since the Schwarzschild $\mathrm{BH}$ frequencies scales as $M \omega=$ const. For the grid spacing, we have used $h=10^{-2} m$ throughout. In what follows, we have used the notation $\omega^{D O M}$ for the dominant mode, $\omega^{S E C}$ for the first overtone and, whenever applicable, $\omega^{T E R}$ for the second overtone. We have also listed the quantities $\delta \omega^{i}, i=D O M, S E C, T E R$, which are the estimated errors for the corresponding frequency values. Such errors were computed using the method described in detail in the Appendix.

Blank spaces, wherever they appear, indicate lack of reliable data for the mode in question.

Before continuing, a few remarks are due: as expected, the scalar field oscillated very little for $\ell=0$ compared to higher $\ell$ values, so we had to remove its power-law tail numerically (see corresponding note in the Appendix) to get a clearer picture of the corresponding oscillations and then compute its first overtone. The second overtone was not clearly visible for $\ell<6$, and even so only for $\ell=8$ and higher we could perform minimally acceptable fittings on it.

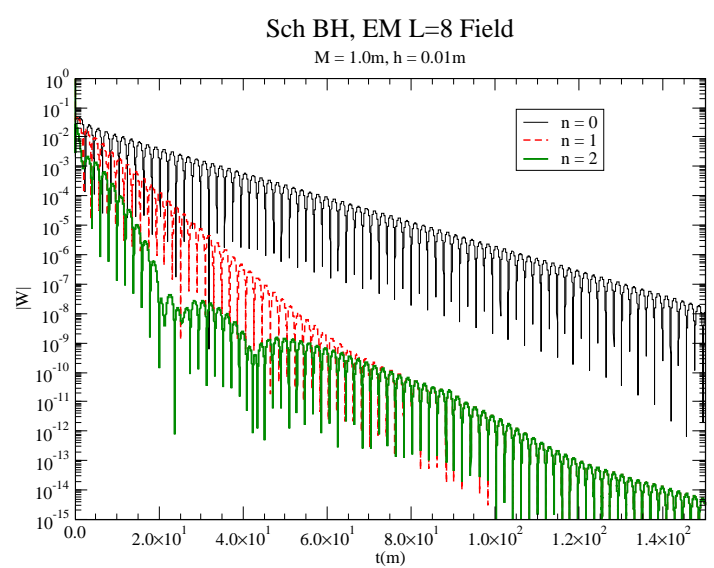

FIG. 1: Fundamental mode and first overtone, for the electromagnetic $\ell=8$ field. The second overtone appears very clearly..

The Figs. (1) and (2) show that the second overtone appears clearly only for very high $\ell(\ell>6)$, and it is the reason why we have blank spaces for this overtone in tables (I) and (II).

We have compared our data to those generated via 6th-order WKB computations, from [5] and 3rd-order WKB from [6]. 
TABLE I: Frequencies for the Schwarzschild BH of $M=1.0$ in the case of a scalar field, for different $\ell$ values.

\begin{tabular}{|c|c|c|c|c|}
\hline$\ell$ & $\omega_{D O M}$ & $\delta \omega_{D O M}$ & $\omega_{\text {SEC }}$ & $\omega_{\text {TER }}$ \\
\hline \hline 0 & $0.107306-0.103929 i$ & - & - & - \\
\hline 1 & $0.292939-0.0976663 i$ & - & - & - \\
\hline 2 & $0.483644-0.0967590 i$ & $3 E-7$ & $0.470-0.300 i$ & - \\
\hline 3 & $0.675367-0.0964997 i$ & $2 E-7$ & $0.667-0.288 i$ & - \\
\hline 4 & $0.867417-0.0963923 i$ & $2 E-7$ & $0.859-0.287 i$ & - \\
\hline 5 & $1.059614-0.0963337 i$ & $6 E-7$ & $1.050-0.283 i$ & - \\
\hline 6 & $1.251891-0.0963060 i$ & $9 E-8$ & $1.224-0.283 i$ & - \\
\hline 7 & $1.444214-0.0962866 i$ & $7 E-7$ & $1.433-0.282 i$ & - \\
\hline 8 & $1.636565-0.0962724 i$ & $1 E-6$ & $1.629-0.288 i$ & $1.584-0.422 i$ \\
\hline 9 & $1.828959-0.0962639 i$ & $1 E-6$ & $1.822-0.288 i$ & $1.791-0.404 i$ \\
\hline 10 & $2.021329-0.0962568 i$ & $1 E-6$ & $2.014-0.288 i$ & $1.990-0.405 i$ \\
\hline 11 & $2.213730-0.0962522 i$ & $1 E-6$ & $2.207-0.288 i$ & $2.174-0.440 i$ \\
\hline 12 & $2.406139-0.0962487 i$ & $1 E-6$ & $2.400-0.288 i$ & $2.371-0.440 i$ \\
\hline
\end{tabular}

TABLE II: Frequencies for the Schwarzschild BH of $M=1.0$, in the case of an electromagnetic field, for different $\ell$ values.

\begin{tabular}{|c|c|c|c|c|}
\hline$\ell$ & $\omega_{D O M}$ & $\delta \omega_{D O M}$ & $\omega_{S E C}$ & $\omega_{T E R}$ \\
\hline \hline 1 & $0.248229-0.0924905 i$ & - & - & - \\
\hline 2 & $0.457595-0.0950044 i$ & $5 E-8$ & $0.440-0.290 i$ & - \\
\hline 3 & $0.656899-0.0956165 i$ & $2 E-8$ & $0.648-0.286 i$ & - \\
\hline 4 & $0.853096-0.0958605 i$ & $2 E-7$ & $0.844-0.285 i$ & - \\
\hline 5 & $1.047915-0.0959821 i$ & $9 E-8$ & $1.039-0.282 i$ & - \\
\hline 6 & $1.242000-0.0960523 i$ & $9 E-8$ & $1.232-0.282 i$ & - \\
\hline 7 & $1.435647-0.0960959 i$ & $7 E-7$ & $1.424-0.281 i$ & - \\
\hline 8 & $1.629012-0.0961250 i$ & $6 E-8$ & $1.621-0.288 i$ & $1.577-0.425 i$ \\
\hline 9 & $1.822180-0.0961452 i$ & $1 E-6$ & $1.815-0.288 i$ & $1.788-0.407 i$ \\
\hline 10 & $2.015214-0.0961596 i$ & $1 E-6$ & $2.008-0.288 i$ & $1.984-0.410 i$ \\
\hline 11 & $2.208148-0.0961714 i$ & $2 E-6$ & $2.199-0.285 i$ & $2.164-0.442 i$ \\
\hline 12 & $2.401004-0.0961800 i$ & $1 E-6$ & $2.395-0.288 i$ & $2.365-0.427 i$ \\
\hline
\end{tabular}

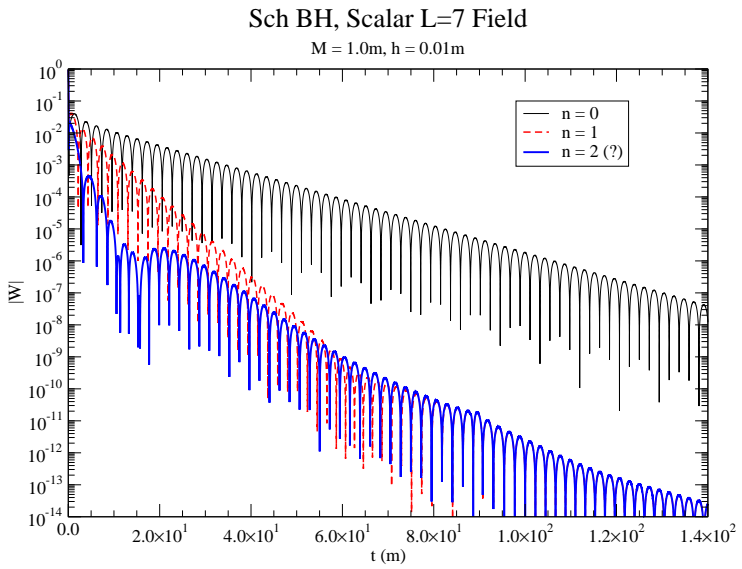

FIG. 2: Fundamental mode and first overtone, for the scalar $\ell=7$ field. The second overtone also appears, though not in a very evident manner.

When it comes to notation, we have employed $\ell$ for the multipole index (as before), $n$ for the overtone index ( $n=0$ for the fundamental, $n=1$ for the first overtone, and so on). $\omega_{N U M}$ refers to our numerical data, $\omega_{W K B 1}$ refers to the WKB data from paper [5] and $\omega_{W K B 2}$, to paper [6]. We were also able to compare our data to a few data compiled two decades ago by E.W. Leaver, who employed yet another method based on continuous fraction equations. See [7], [8] and references therein.

These comparison tables leave no room for doubts when it comes to the fundamental mode, $n=0$. For all fields and $\ell$-values, the data from our numerical simulations and those from the 6th-order WKB method showed a high-degree agreement, with differences between 1 part in $10^{4}$ and 1 part in $10^{5}$, for both $\operatorname{Re}(\omega)$ and $\operatorname{Im}(\omega)$, especially for higher $\ell$-values. When it comes to the first overtone, for all fields under study, the agreement between both sets of data was not so impressive, hovering around $1-2 \%$ for $\operatorname{Re}(\omega)$ and $2-3 \%$ for $\operatorname{Im}(\omega)$ for low $\ell$ (typically up to $\ell=6$ ) and improving for higher $\ell$, to a few parts in 1000 for $\operatorname{Re}(\omega)$ and around $0.5 \%$ or so for $\operatorname{Im}(\omega)$. The second overtone showed much higher discrepancies, especially for $\operatorname{Im}(\omega)$, with differences around $15 \%$ is some cases, while for $\operatorname{Re}(\omega)$ this difference usually hovers around $1-2 \%$ (with two exceptions for $\ell=8$, when it was much bigger - almost $8 \%$ for the axial field).

As for the data from Leaver [7],[8], we have, for the gravitational axial perturbation and $\ell=2, \omega=0.373672-$ $0.088962 i$ when $n=0$ and $\omega=0.346711-0.273915 i$ when $n=1$. For $n=0$ this matches very well with our result, $\omega=0.37367-0.08896 i$. For $n=1$, the agreement is not so good, because we got $\omega=0.352-0.272 i$ but Leaver's re- 
TABLE III: Frequencies for the Schwarzschild BH of mass $M=1.0$, in the case of an axial field, for different $\ell$ values.

\begin{tabular}{|c|c|c|c|c|}
\hline$\ell$ & $\omega_{D O M}$ & $\delta \omega_{D O M}$ & $\omega_{\text {SEC }}$ & $\omega_{\text {TER }}$ \\
\hline \hline 2 & $0.37367-0.08896 i$ & $6 E-6$ & $0.352-0.271 i$ & - \\
\hline 3 & $0.599444-0.0927031 i$ & $5 E-9$ & $0.586-0.278 i$ & - \\
\hline 4 & $0.809180-0.0941643 i$ & $4 E-8$ & $0.797-0.279 i$ & - \\
\hline 5 & $1.012297-0.0948713 i$ & $3 E-7$ & $1.002-0.279 i$ & - \\
\hline 6 & $1.212013-0.0952667 i$ & $2 E-7$ & $1.203-0.286 i$ & - \\
\hline 7 & $1.409741-0.0955106 i$ & $2 E-7$ & $1.401-0.286 i$ & $1.361-0.432 i$ \\
\hline 8 & $1.606202-0.0956724 i$ & $3 E-7$ & $1.594-0.280 i$ & $1.474-0.515 i$ \\
\hline 9 & $1.801801-0.0957836 i$ & $1 E-6$ & $1.795-0.287 i$ & $1.770-0.411 i$ \\
\hline 10 & $1.996796-0.0958649 i$ & $2 E-7$ & $1.990-0.286 i$ & $1.967-0.408 i$ \\
\hline 11 & $2.191345-0.0959263 i$ & $2 E-7$ & $2.188-0.287 i$ & $2.154-0.415 i$ \\
\hline 12 & $2.385555-0.0959724 i$ & $2 E-7$ & $2.379-0.287 i$ & $2.353-0.446 i$ \\
\hline
\end{tabular}

TABLE IV: Frequencies for the Schwarzschild BH of $M=1.0$, in the case of a scalar field, for different $\ell$ and $n$ values, and their comparison to WKB data.

\begin{tabular}{|c|c|c|c|c|}
\hline$\ell$ & $n$ & $\omega_{N U M}$ & $\omega_{W K B 1}$ & $\omega_{W K B 2}$ \\
\hline \hline 0 & 0 & $0.107306-0.103929 i$ & $0.1105-0.1008 i$ & $0.1046-0.1152 i$ \\
\hline 1 & 0 & $0.292939-0.0976663 i$ & $0.2929-0.0977 i$ & $0.2911-0.0980 i$ \\
\hline 1 & 1 & - & $0.264-0.307 i$ & $0.2622-0.3074 i$ \\
\hline 2 & 0 & $0.483644-0.0967590 i$ & $0.4836-0.0968 i$ & $0.4832-0.0968 i$ \\
\hline 2 & 1 & $0.470-0.300 i$ & $0.4638-0.2956 i$ & $0.4632-0.2958 i$ \\
\hline 2 & 2 & - & $0.4317-0.5034 i$ & $0.4317-0.5034 i$ \\
\hline 3 & 0 & $0.675367-0.0964997 i$ & $0.675366-0.0965006 i$ & - \\
\hline 3 & 1 & $0.667-0.288 i$ & $0.660671-0.292288 i$ & - \\
\hline 4 & 0 & $0.867417-0.0963923 i$ & $0.867416-0.0963919 i$ & - \\
\hline 4 & 1 & $0.859-0.287 i$ & $0.855808-0.290877 i$ & - \\
\hline 5 & 0 & $1.059614-0.0963337 i$ & $1.05961-0.0963368 i$ & - \\
\hline 5 & 1 & $1.050-0.283 i$ & $1.05004-0.290154 i$ & - \\
\hline 6 & 0 & $1.251891-0.0963060 i$ & $1.25189-0.0963051 i$ & - \\
\hline 6 & 1 & $1.224-0.283 i$ & $1.24375-0.289736 i$ & - \\
\hline 7 & 0 & $1.444214-0.0962866 i$ & $1.44421-0.0962852 i$ & - \\
\hline 7 & 1 & $1.433-0.282 i$ & $1.43714-0.289473 i$ & - \\
\hline 8 & 0 & $1.636565-0.0962724 i$ & $1.63656-0.0962719 i$ & - \\
\hline 8 & 1 & $1.629-0.288 i$ & $1.63031-0.289297 i$ & \\
\hline 8 & 2 & $1.584-0.422 i$ & $1.61797-0.483757 i$ & \\
\hline 9 & 0 & $1.828939-0.0962639 i$ & $1.82893-0.0962626 i$ & - \\
\hline 9 & 1 & $1.822-0.288 i$ & $1.82333-0.289173 i i$ & \\
\hline 9 & 2 & $1.791-0.404 i$ & $1.81225-0.483235 i$ & \\
\hline 10 & 0 & $2.021329-0.0962568 i$ & $2.02132-0.0962558 i$ & - \\
\hline 10 & 1 & $2.014-0.288 i$ & $2.01625-0.289083 i$ & \\
\hline 10 & 2 & $1.990-0.405 i$ & $2.0062-0.482854 i$ & \\
\hline 11 & 0 & $2.213730-0.0962522 i$ & $2.21372-0.0962507 i$ & - \\
\hline 11 & 1 & $2.207-0.288 i$ & $2.20909-0.289015 i$ & \\
\hline 11 & 2 & $2.174-0.417 i$ & $2.19989-0.482567 i$ & \\
\hline 12 & 0 & $2.406139-0.0962487 i$ & $2.40613-0.0962467 i$ & - \\
\hline 12 & 1 & $2.400-0.288 i$ & $2.40186-0.288963 i$ & \\
\hline 12 & 2 & $2.371-0.440 i$ & $2.39338-0.482347 i$ & \\
\hline & & & & \\
\hline
\end{tabular}

sult agrees very well with the 6th-order WKB result on both cases. When we looked at the $\ell=3$ case for the same perturbation, Leaver's results were $\omega=0.599443-0.092703 i$ when $n=0$ and $\omega=0.582644-0.281248 i$ when $n=1$. Our results were $0.599444-0.0927031 i$ and $0.587-0.278 i$, respectively. Again, the agreement between Leaver's results and ours is excellent for the $n=0$ mode, but not so good for $n=1$ : ours were higher by about $1 \%$ for both $\operatorname{Re}(\omega)$ and $\operatorname{Im}(\omega)$. And the 6th-order WKB and Leaver's results again agreed very well on both cases (see Table VI). For the $\ell=4$ case, Leaver's figures were $\omega=0.80918-0.094165 i$ $(n=0)$ and $\omega=0.79663-0.28433 i(n=1)$. Our data were $\omega=0.809180-0.0941643 i(n=0)$ and $\omega=0.797-0.279 i$ $(n=1)$, this time showing a better agreement for $\operatorname{Re}(\omega)$, at least. We were not able to check Leaver's results for higher $\ell$ values, so we cannot yet say whether the discrepancies between our data and Leaver's data will remain for $n=1$ and higher when $\ell$ icreases beyond 5. But for the $n=0$ modes the 
TABLE V: Frequencies for the Schwarzschild BH of $M=1.0$, in the case of an electromagnetic field, for different $\ell$ and $n$ values, and their comparison to WKB data.

\begin{tabular}{|c|c|c|c|c|}
\hline$\ell$ & $n$ & $\omega_{N U M}$ & $\omega_{W K B 1}$ & $\omega_{W K B 2}$ \\
\hline \hline 1 & 0 & $0.248229-0.0924905 i$ & $0.2482-0.0926 i$ & $0.2459-0.0931 i$ \\
\hline 1 & 1 & - & $0.2143-0.2941 i$ & $0.2113-0.2958 i$ \\
\hline 2 & 0 & $0.457595-0.0950044 i$ & $0.4576-0.0950 i$ & $0.4571-0.0951 i$ \\
\hline 2 & 1 & $0.440-0.290 i$ & $0.4365-0.2907 i$ & $0.4358-0.2910 i$ \\
\hline 2 & 2 & - & $0.4009-0.5017 i$ & $0.4023-0.4959 i$ \\
\hline 3 & 0 & $0.656899-0.0956165 i$ & $0.6569-0.0956 i$ & $0.6567-0.0951 i$ \\
\hline 3 & 1 & $0.648-0.286 i$ & $0.6417-0.2897 i$ & $0.6415-0.2898 i$ \\
\hline 3 & 2 & - & $0.6138-0.4921 i$ & $0.6151-0.4901 i$ \\
\hline 3 & 3 & - & $0.5814-0.6955 i$ & $0.5814-0.6955 i$ \\
\hline 4 & 0 & $0.853096-0.0958605 i$ & $0.853095-0.0958601 i$ & - \\
\hline 4 & 1 & $0.844-0.285 i$ & $0.841267-0.289315 i$ & - \\
\hline 5 & 0 & $1.047915-0.0959821 i$ & $1.04791-0.095981 i$ & - \\
\hline 5 & 1 & $1.039-0.282 i$ & $1.03822-0.289104 i$ & - \\
\hline 6 & 0 & $1.242000-0.0960523 i$ & $1.242-0.0960512 i$ & - \\
\hline 6 & 1 & $1.232-0.282 i$ & $1.23379-0.288982 i$ & - \\
\hline 7 & 0 & $1.435647-0.0960959 i$ & $1.43564-0.0960947 i$ & - \\
\hline 7 & 1 & $1.424-0.281 i$ & $1.42852-0.288906 i$ & - \\
\hline 8 & 0 & $1.629012-0.0961250 i$ & $1.629-0.0961236 i$ & - \\
\hline 8 & 1 & $1.621-0.288 i$ & $1.62272-0.288855 i$ & - \\
\hline 8 & 2 & $1.577-0.425 i$ & $1.61032-0.483028 i$ & - \\
\hline 9 & 0 & $1.822180-0.0961452 i$ & $1.82217-0.0961439 i$ & - \\
\hline 9 & 1 & $1.815-0.288 i$ & $1.81655-0.288819 i$ & - \\
\hline 9 & 2 & $1.788-0.407 i$ & $1.80542-0.48265 i$ & - \\
\hline 10 & 0 & $2.015214-0.0961596 i$ & $2.01521-0.0961587 i$ & - \\
\hline 10 & 1 & $2.006-0.288 i$ & $2.01012-0.288793 i$ & - \\
\hline 10 & 2 & $1.984-0.410 i$ & $2.00003-0.482374 i$ & - \\
\hline 11 & 0 & $2.208148-0.0961714 i$ & $2.20814-0.0961697 i$ & - \\
\hline 11 & 1 & $2.199-0.285 i$ & $2.20349-0.288773 i$ & - \\
\hline 11 & 2 & $2.161-0.442 i$ & $2.19426-0.482167 i$ & - \\
\hline 12 & 0 & $2.401004-0.096180 i$ & $2.40099-0.0961782 i$ & - \\
\hline 12 & 1 & $2.395-0.288 i$ & $2.39672-0.288758 i$ & - \\
\hline 12 & 2 & $2.365-0.427 i$ & $2.38822-0.482007 i$ & - \\
\hline & & & & \\
\hline
\end{tabular}

agreement between both sets of results seemed to be beyond any doubt to us.

In short, from the few data we have from Leaver's work, we might say that Leaver's data seem to be more precise for the Schwarzschild case. Our method, however, may be useful when the perturbative potentials are too awkward or complicated to conform to an analytic or semi-analytic method of computing quasinormal frequencies, so that direct numerical integration remains the sole option for that goal.

A brief remark is due on the scalar $\ell=0$ case. As we had already pointed out in this text, we had to subtract the powerlaw tail from the original field, so that we could have a sufficent number of oscillations to render any frequency fitting meaningful. For that particular case, however, the agreement between our data and 6th-order WKB was not perfect, since we got $\omega=0.107306-0.103929 i$, while the 6th-order WKB result was $\omega=0.1106-0.1008 i$, that is, a discrepancy of $3 \%$ for $\operatorname{Re}(\omega)$ and $7 \%$ for $\operatorname{Im}(\omega)$. See Fig. (3).

\section{APPENDIX A: ON THE NUMERICAL METHOD FOR OBTAINING THE WAVE PROFILES}

We provide here a brief sketch of the method employed to find the wave profiles - let the grid in which the method operates be called $x-t$ grid - from which we have later extracted the dominant mode and the overtones - the latter theme will be thoroughly treated in the next section.

The $x-t$ grid provides direct integration in the time domain, using a numerical method which is second-order in time (denoted $t$ ) and fourth-order in the spatial coordinate (the socalled tortoise coordinate in a Schwarzschild spacetime, denoted $x$ ). Let $\Psi(x, t)$ represent the field (whatever its nature) at a given $x$ and a given $t$. Let $\delta x$ and $\delta t$ represent the spacing along $x$ and $t$, respectively. Both $\delta x$ and $\delta t$ are constant, though $\delta t<\delta x$ for reasons we shall see immediately. Hence, 
TABLE VI: Frequencies for the Schwarzschild BH of mass $M=1.0$, in the case of an axial field, for different $\ell$ and $n$ values, and their comparison to WKB data.

\begin{tabular}{|c|c|c|c|c|}
\hline$\ell$ & $n$ & $\omega_{N U M}$ & $\omega_{W K B 1}$ & $\omega_{W K B 2}$ \\
\hline \hline 2 & 0 & $0.37367-0.08896 i$ & $0.373691-0.088891 i$ & $0.3732-0.0892 i$ \\
\hline 2 & 1 & $0.352-0.272 i$ & $0.346297-0.27348 i$ & $0.3460-0.2749 i$ \\
\hline 2 & 2 & - & $0.2985-0.4776 i$ & $0.29852-0.47756 i$ \\
\hline 3 & 0 & $0.599444-0.0927031 i$ & $0.599443-0.0927025 i$ & $0.5993-0.0927 i$ \\
\hline 3 & 1 & $0.587-0.278 i$ & $0.582642-0.28129 i$ & $0.5824-0.2814 i$ \\
\hline 3 & 2 & - & $0.551594-0.479047 i$ & $0.5532-0.4767 i$ \\
\hline 4 & 0 & $0.809180-0.0941643 i$ & $0.809178-0.0941641 i$ & $0.8091-0.0942 i$ \\
\hline 4 & 1 & $0.797-0.279 i$ & $0.796631-0.284334 i$ & $0.7965-0.2844 i$ \\
\hline 4 & 2 & - & $0.7727-0.4799 i$ & $0.772695-0.4799 i$ \\
\hline 5 & 0 & $1.012297-0.0948713 i$ & $1.0123-0.0948706 i$ & - \\
\hline 5 & 1 & $1.002-0.279 i$ & $1.00222-0.285817 i$ & - \\
\hline 6 & 0 & $1.212013-0.0952667 i$ & $1.21201-0.0952659 i$ & - \\
\hline 6 & 1 & $1.203-0.286 i$ & $1.20357-0.28665 i$ & - \\
\hline 7 & 0 & $1.409741-0.0955106 i$ & $1.40974-0.0955096 i$ & - \\
\hline 7 & 1 & $1.401-0.286 i$ & $1.40247-0.287164 i$ & - \\
\hline 7 & 2 & $1.361-0.432 i$ & $1.38818-0.480709 i$ & - \\
\hline 8 & 0 & $1.606202-0.0956724 i$ & $1.60619-0.0956707 i$ & - \\
\hline 8 & 1 & $1.594-0.280 i$ & $1.59981-0.287504 i$ & - \\
\hline 8 & 2 & $1.474-0.515 i$ & $1.58721-0.480804 i$ & - \\
\hline 9 & 0 & $1.801801-0.095784 i$ & $1.80179-0.0957828 i$ & - \\
\hline 9 & 1 & $1.795-0.288 i$ & $1.7961-0.287741 i$ & - \\
\hline 9 & 2 & $1.770-0.440 i$ & $1.78483-0.48087 i$ & - \\
\hline 10 & 0 & $1.996796-0.0958649 i$ & $1.99679-0.0958639 i$ & - \\
\hline 10 & 1 & $1.990-0.286 i$ & $1.99165-0.287912 i$ & - \\
\hline 10 & 2 & $1.967-0.408 i$ & $1.98145-0.48087 i$ & - \\
\hline 11 & 0 & $2.191345-0.0959263 i$ & $2.19133-0.0959245 i$ & - \\
\hline 11 & 1 & $2.185-0.287 i$ & $2.18665-0.28804 i$ & - \\
\hline 11 & 2 & $2.154-0.415 i$ & $2.17734-0.480952 i$ & - \\
\hline 12 & 0 & $2.385555-0.0959724 i$ & $2.38554-0.095971 i$ & - \\
\hline 12 & 1 & $2.379-0.287 i$ & $2.38124-0.288138 i$ & - \\
\hline 12 & 2 & $2.353-0.446 i$ & $2.37268-0.480979 i$ & - \\
\hline & & & & - \\
\hline
\end{tabular}

one may evolve the field $\Psi$ in time via

$$
\begin{aligned}
& \Psi\left(t_{0}+\delta t, x_{0}\right)= \\
- & \Psi\left(t_{0}-\delta t, x_{0}\right)+\left(2-\delta^{2} x V\left(x_{0}\right)-\frac{5 \delta t^{2}}{2 \delta x^{2}}\right) \Psi\left(t_{0}, x_{0}\right)+ \\
+ & \frac{4 \delta t^{2}\left[\Psi\left(t_{0}, x_{0}+\delta x\right)+\Psi\left(t_{0}, x_{0}-\delta x\right)\right]}{3 \delta x^{2}}- \\
- & \frac{\delta t^{2}\left[\Psi\left(t_{0}, x_{0}-2 \delta x\right)+\Psi\left(t_{0}, x_{0}+2 \delta x\right)\right]}{12 \delta x^{2}}
\end{aligned}
$$

In the prescription (A1), one computes the field $\Psi$ at $(x, t)=$ $\left(x_{0}, t_{0}+\delta t\right)$ from the values of the field at 5 different points at the instant $t_{0}$ and from the value of the field in one point at the instant $t_{0}-d t$. At the initial instant $t_{\text {ini }}$, one specifies not only $\Psi$, but also $\frac{\partial \Psi}{\partial t}$, for the whole spatial range $x_{i n i}<x<x_{\text {fin }}$. The very structure of (A1) causes $x_{\max }$ to increase by $2 \delta x$ and $x_{\text {min }}$ to decrease by the same amount - the algorithm develops in time like an inverted cone - and the integration stopped for a predetermined value of $t$ (or time steps). An illustration of the method is provided in Fig. (A).

A point of paramount importance is to be stressed for the current method: the time spacing $\delta_{t}$ must be always smaller in magnitude than the spacing in $x, \delta_{x}$. That is, if $m=\frac{\delta_{t}}{\delta_{x}}<1$.
This is necessary to preserve numerical stability. This $m$ is called mesh ratio.

As for the initial conditions in use, they make little difference in the final result, since we are interested in the quasinormal ringdown of the fields, which do not depend on the initial condition. We have used an initial Gaussian pulse of unit magnitude centered at $x=0$, due to its ease of implementation.

\section{APPENDIX B: FINDING THE OVERTONES NUMERICALLY}

Having presented the $x-t$ grid to find the wave profile, we can turn to the problem of extracting the dominant mode and its overtones from them. Hence, in this section, we present a thorough explanation on the method of extracting the dominant mode, the secondary (and, whenever applicable, the tertiary) mode: we have taken the first (or dominant) mode $(n=0)$ from the original wave profile and then performed an oscillatory fitting on it, in order to extract the data on its oscillatory frequency $\omega_{R}$, amplitude $A$ and decay rate $\omega_{I}$. A question which rises naturally is: given the original wave profile, 


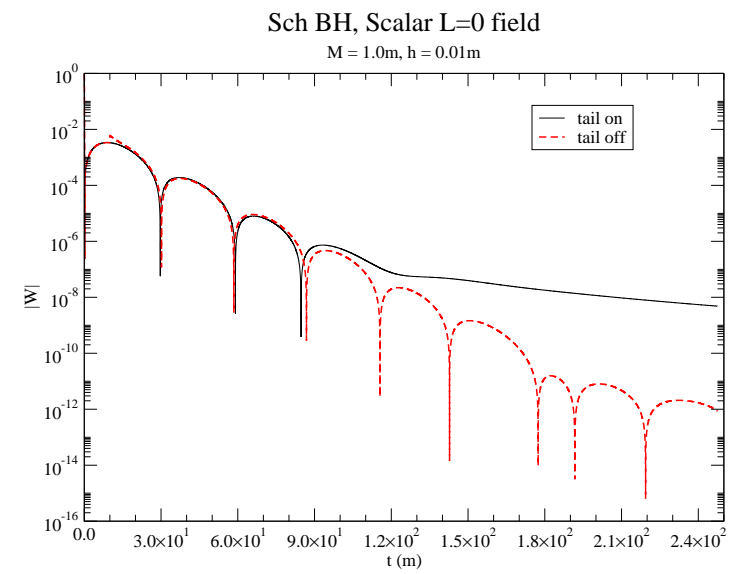

FIG. 3: Fundamental mode with and without tail, for the scalar $\ell=0$ field. Notice how the oscillations are masked by the power-law tail.

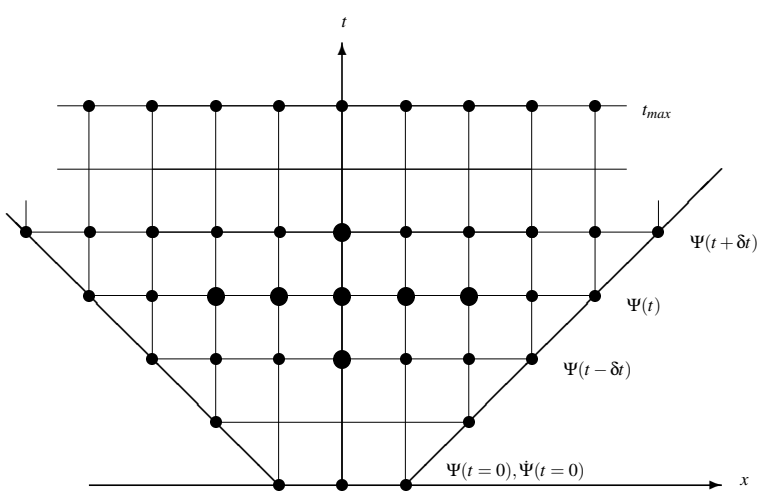

FIG. 4: The $x-t$ grid.

how do we recognize the dominant mode, since the overtones also contribute to the wave profile ?

The answer is that we expect the overtones to decay much faster than the dominant mode, so that their contribution shows up at the early stages of the waveform (transient part and the early ringdown phase). Hence the late-time ringdown phase is completely dominated by this $n=0$ mode, and the aforementioned quantities $A, \omega_{I}, \omega_{R}$ are taken at this phase. The reader, upon looking again at the Figs. (1) and (2), will be convinced that this is indeed the case.

These data on the $n=0$ mode were taken with the maximum number of significant digits possible (usually 8 or 9), and we subtracted the fitted function from the original wave profile. That fitted function had the form

$$
\Psi_{0}=A \exp \left(i \omega_{R}^{0} t\right) \exp \left(-\omega_{I}^{0} t\right)
$$

so that we could see the remainder of this operation.

In all cases (except for the $\ell=0,1$ scalar and $\ell=1 \mathrm{EM}$ field), after performing the subtraction above, we could find a remainder of the form

$$
\Psi_{R}=B \exp \left(i \omega_{R}^{1} t\right) \exp \left(-\omega_{I}^{1} t\right)+\Delta,
$$

in which $\omega_{1} \neq \omega_{0}$, characterizing a secondary mode (the first overtone), and with similar amplitudes $(A \approx B)$. The term $\Delta$ is just the $\Psi_{0}$ term, but with a much reduced amplitude, indicating that the fitting operation has its precision limitations. This can be seen in the form of parallel curve envelopes in the Figs. (1) and (2), for example. Needless to say, we picked the $n=1$ mode fitting data at the end of its own quasinormal ringing phase, for the same reasons outlined above for the $n=0$ mode.

A similar procedure was adopted to find the second overtone, this time applying the oscillatory fitting to the first overtone, in which a new remainder similar to that seen in (B2) was seen. In this latter case, however, only the very high $\ell$-values could yield any valuable result when an oscillatory fitting was applied to it. This is directly related to the degree of precision to which we could find the amplitude and the frequencies $\omega_{R}$ and $\omega_{I}$ : since the number of significant figures for them was around 3. Less significant figures translate into greater errors in the subtraction process to find the tertiary mode, which in turn translates into somewhat blurred wave profiles for that tertiary mode. Another difficulty involved in finding it is that it decays much faster than the $n=0$ and $n=1$, which means it is available for extraction only at the very early stages of the original waveform. This very reduced time interval for mode extraction compromises the oscillatory fitting quality and is responsible for the $n=1$ mode's much lower number of significant figures when compared to the $n=0$ mode, since the $n=2$ mode is used to estimate the $n=1$ mode's fitting precision.

The fact that in some cases we cannot see the $n=1$ mode can be easily explained: these cases correspond to very low values for $\ell$, namely $\ell=0,1$, for which very few oscillations appear even for the dominant mode, thus rendering it very difficult, if not impracticable, to extract an overtone from the corresponding wave profiles. The $n=2$ mode, in turn, is usually only detectable for higher values of $\ell(\ell>6)$. We are still figuring out why this happens.

Now it is the time to estimate the precision of the first fitting. As we can see again in the aforementioned figures, there is a given time $t_{c}$ for which the secondary mode and the dominant mode are roughly equal in magnitude, that is

$$
B \exp \left(i \omega_{R}^{1} t\right) \exp \left(-\omega_{I}^{1} t\right) \approx \delta\left(A \exp \left(i \omega_{R}^{0} t\right) \exp \left(-\omega_{I} t\right)\right) .
$$

The variation of the dominant mode can be expressed as

$$
\begin{aligned}
& \delta\left(A \exp \left(i \omega_{R}^{0} t\right) \exp \left(-\omega_{I} t\right)\right)=(\delta A) \exp \left(i \omega_{R}^{0} t\right) \exp \left(-\omega_{I} t\right)+ \\
+ & A i \exp \left(i \omega_{R} t\right) \exp \left(-\omega_{I} t\right)\left[\delta \omega_{R}^{0}+i \delta \omega_{I}^{0}\right] t .
\end{aligned}
$$

In what follows, we assume $\delta A$ to be much smaller than $A$. Such approximation implies

$$
B \exp \left(i \omega_{R}^{1} t\right) \exp \left(-\omega_{I}^{1} t\right) \approx A i t \delta \omega \exp \left(i \omega_{R}^{0}\right) \exp \left(-\omega_{I}^{1} t\right),
$$

in which we have used $\delta \omega=\delta \omega_{R}^{0}+i \delta \omega_{I}^{0}$. Working with the moduli of the quantities above, we arrive at

$$
|\delta \omega| \approx\left|\frac{B}{A}\right| \frac{\exp \left(-\left[\omega_{I}^{1}-\omega_{I}^{0}\right]\right) t}{t} .
$$


Upon substituting $t=t_{c}$ and noting that $|A| \approx|B|$, we arrive at

$$
|\delta \omega| \approx \frac{\exp \left(-\left[\omega_{I}^{1}-\omega_{I}^{0}\right]\right) t_{c}}{t_{c}} .
$$

Such an estimate was done for each field and for each $\ell$-value, and $|\delta \omega|$ was compared to $|\omega|$, so as to determine the approximate degree of precision in the computation of the dominant mode frequency. A similar estimate can also be done, in principle, for the first overtone fitting precision. We have determined the ratio $m=\left|\frac{\delta \omega}{\omega}\right|$ - for the $n=0$ mode - to be smaller than $10^{-6}$, in all cases we have dealt with, except for the axial $\ell=2$ case, for which $m \approx 10^{-5}$ (hence its smaller number of significant figures).

A final remark is needed for the scalar field, in the $\ell=0$ case. Since this particular field decays as a power-law tail quite soon in the time domain (compared to higher $\ell$ fields), we had to perform a power-law fitting (the classical powerlaw tail of the fields evolving in a Schwarzschild spacetime) on its tail and then subtract it from the original mode, therefore unveiling oscillations decaying beneath the tail, as already seen in Fig. (3). The numerical unveiling of its first overtone was then tried in the same way as in all the remaining fields, though we failed to get a clear picture of it, for the reasons described earlier in this Appendix.
[1] F. Pretorius, Phys. Rev. Lett. 95, 121101 (2005).

[2] T. Regge and J. A. Wheeler, Phys. Rev. 108, 1063 (1957); S. Chandrasekhar, The Mathematical Theory of Black Holes, (Oxford University Press, Oxford, 1983); K. D. Kokkotas and B. G. Schmidt, Living Rev. Relativity 2 (1999).

[3] O.D. Aguiar et al., Class. Quant. Grav. 23, S239 (2006).

[4] R. Price, Phys. Rev. D 5, 2419 (1972); C. Gundlach, R. Price, and J. Pullin, Phys. Rev. D 49, 883 (1994); V. Cardoso and J. P. S. Lemos, Phys. Rev. D 64, 084017 (2001); Bin Wang, Chi-Yong Lin, and Elcio Abdalla Phys. Lett. B 481 (2000) 79; Bin Wang,
C. Molina, and Elcio Abdalla Phys. Rev. D 63, 084001 (2001); C. Molina, D. Giugno, E. Abdalla, and A. Saa, Phys. Rev. D 69, 104013 (2004); R. A. Konoplya, A. V. Zhidenko Phys. Lett. B 609, 377 (2005); E. Abdalla, B. Cuadros-Melgar, A. B. Pavan, and C. Molina Nucl. Phys. B 752, 40 (2006).

[5] R. A. Konoplya, J. Phys. Stud. 8, No. 1, 93 (2004).

[6] S. Iyer, Phys. Rev. D 35, 12, 3632 (1987).

[7] E. W. Leaver, Proc. R. Soc. London Ser. A 402, 285 (1985).

[8] E. W. Leaver, Phys. Rev. D 34, 384 (1986). 